Conference Paper

\title{
I-OT.Net As Internet of Things (IoT) Cloud in Internet-Based Control System Applications
}

\author{
Basuki Rahmat ${ }^{1 *}$, Ida Retno Moeljani², Bhakti Wisnu Widjajani.2, Sudiyarto², Harianto \\ ${ }^{1}$ Informatics Department, Computer Science Faculty, Universitas Pembangunan Nasional “Veteran” Jawa \\ Timur, Indonesia \\ ${ }^{2}$ Agriculture Faculty, Universitas Pembangunan Nasional “Veteran” Jawa Timur, Indonesia \\ ${ }^{3}$ Department of Computer Engineering, Technology and Informatics Faculty, Universitas Dinamika, \\ Surabaya, Indonesia
}

*Corresponding author:

E-mail:

basukirahmat.if@upnjatim.ac.id

\begin{abstract}
With the rapid development and deep application and collaboration of new concepts and technologies brought by the Internet of Things (IoT) and cloud computing around the world, all walks of life are gradually moving towards a smart modern society. This technology has gradually penetrated almost all fields, from simple technology to complex technology. Where the basic system of the Internet of Things consists of 3 things, namely: hardware/physical (things), internet connection, and cloud data center as a place to store or run the application. This paper introduces a new Internet of Things cloud in Indonesia, namely i-ot.net. And examples of its application for Miniature Temperature Control System and Smart Farming are given.
\end{abstract}

Keywords: IoT, internet, things, cloud, and smart

\section{Introduction}

Internet of Things (IoT) is an emerging area in which billions of smart objects are connected using the internet to share data and resources (Chahal et al., 2020). IoT technology allows objects around us to be connected to the internet network. Where every object connected to the internet can be accessed anytime and anywhere. For example, we can remotely turn on and off appliances at home (lights, televisions, stoves, heaters, etc.) as long as the equipment is connected to the IoT cloud and an internet connection is available. In general, the IoT architecture consists of an Application Layer, Middleware Layer, Network Layer, and Physical Layer, as shown in Figure-1 (Ravidas et al., 2019). 


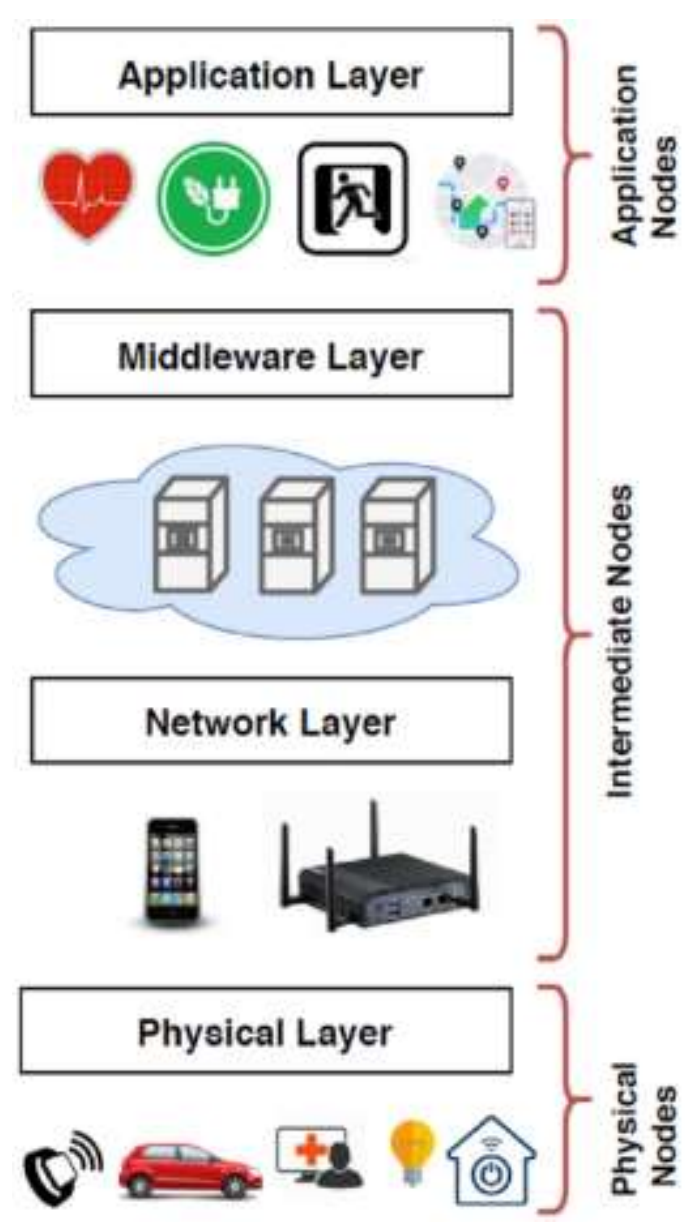

Figure 1. IoT architecture (Ravidas et al., 2019)

Application Layer: aims to provide services to end-users. This layer consists of application nodes that handle application logic as well as data and presentation semantics. This node receives data from the middleware and processes it depending on the requirements of the end-user and the type of service provided. In addition, the application layer includes an Application Programming Interface (API) to facilitate communication with the middleware and user interfaces that end-users use to access services.

Middleware Layer: to ensure connectivity and interoperability in the IoT ecosystem. It consists of intermediate nodes which process the data received from the lower layer and pass it to the application layer.

Network Layer: to support the network and data transfer between nodes. The network layer implements the communication protocols required for data exchange in the IoT ecosystem.

Physical Layer: to characterize the sensing and control capabilities of the IoT system. This layer consists of physical nodes such as sensors and actuators that sense the environment and interact with it in response to user changes or requests. These nodes generate resources (sense data) which are passed to application nodes via the network and middleware layer.

There are many examples of applying IoT technology, a few examples:

- Smart Home (internet-based home security system, can find out the condition of the house and control household appliances and energy consumption via the internet network) (Iqbal et al., 2018; Muralidhara et al., 2020). 
- Smart Farming (internet-based smart farming system, for monitoring and controlling the quality of water and agricultural land as well as crop growth and others via the internet network) (Castañeda-Miranda \& Castaño-Meneses, 2020; Ramli et al., 2020).

- Internet industry (monitoring and control of equipment and processes in the industry) (Huang, 2020; Chen, 2020).

- Health (monitoring a person's health condition) (Mukherjee et al., 2020; Deperlioglu et al., 2020).

- Transportation (management and traffic information) (Feng \& Hu, 2020; Zhang \& Lu, 2020).

- Robotics (formation, control, and integration of internet-based robots) (Guan et al., 2020; Pinheiro et al., 2020).

The basic system of the IoT consists of 3 components, namely:

- Hardware/physical (Things).

- Internet connection, and

- Cloud data center as a place to store or run the application.

Each is shown in Figure 2 (anonymous, 2019).

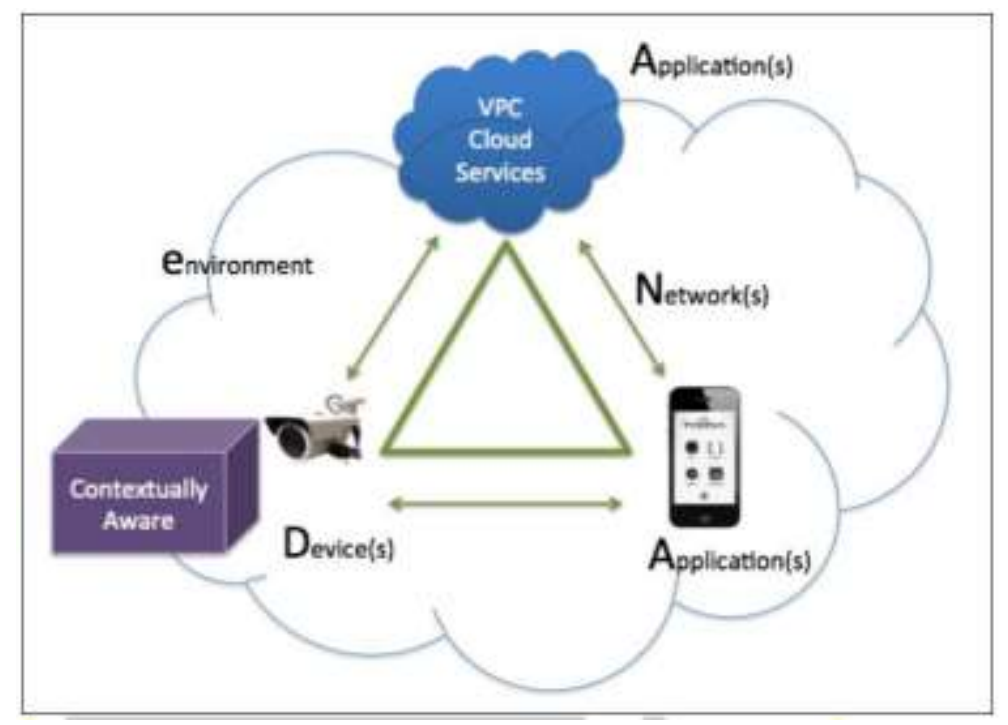

Figure 2. Sistem IoT (anonymous, 2019)

\section{Material and Methods}

As described above, the basic IoT system consists of 3 things, namely: hardware/physical (objects), internet connection, and a cloud data center as a place to store or run applications. This paper introduces a new IoT cloud in Indonesia, namely i-ot.net. And to test its reliability it was tested for Miniature Temperature Control Systems and Smart Farming. To be able to use this IoT Cloud service, please create an account and get the settings needed for remote control of the two plants, via the https://i-ot.net address. Furthermore, the data is used for settings in the microcontroller program. An architectural description of the Miniature Temperature Control System and Smart Farming, as shown in Figure 3 and Figure 4. 


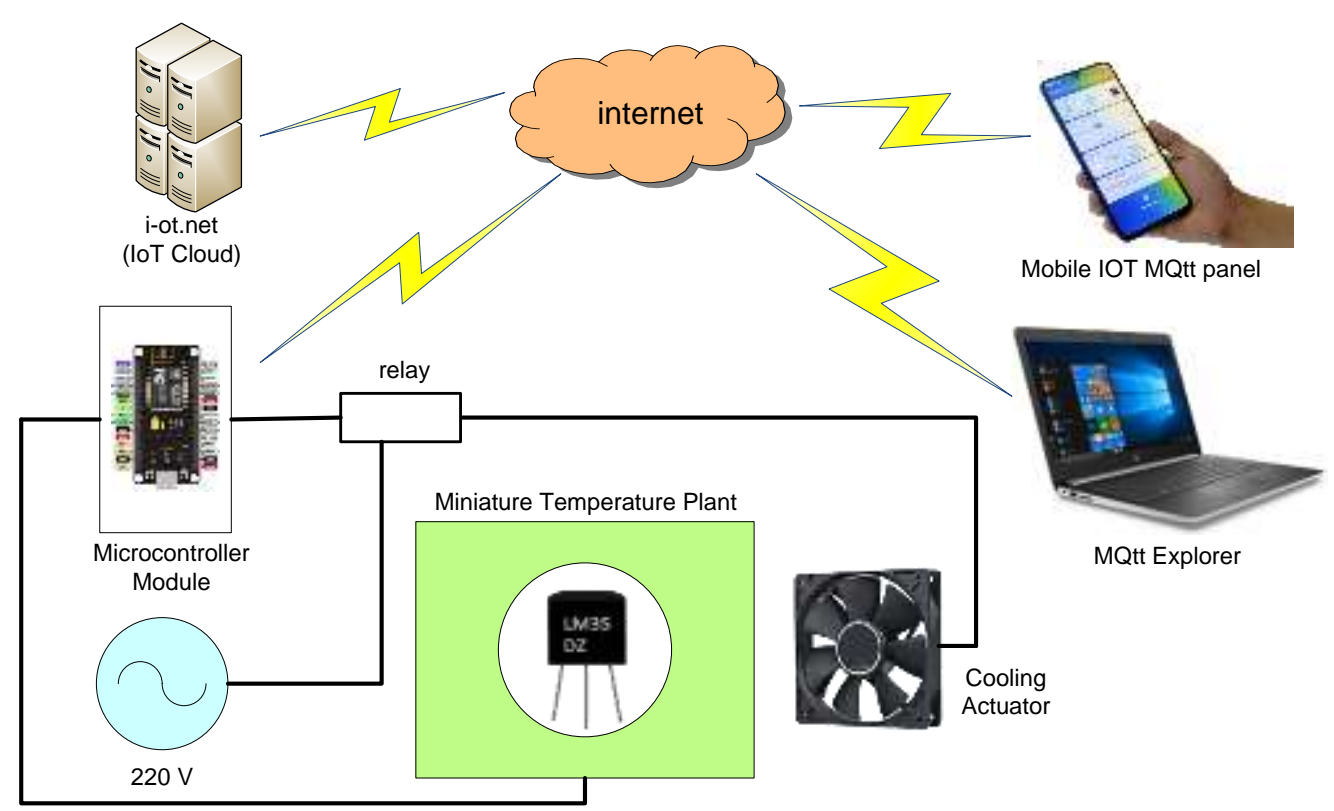

Figure 3. Miniature temperature control system

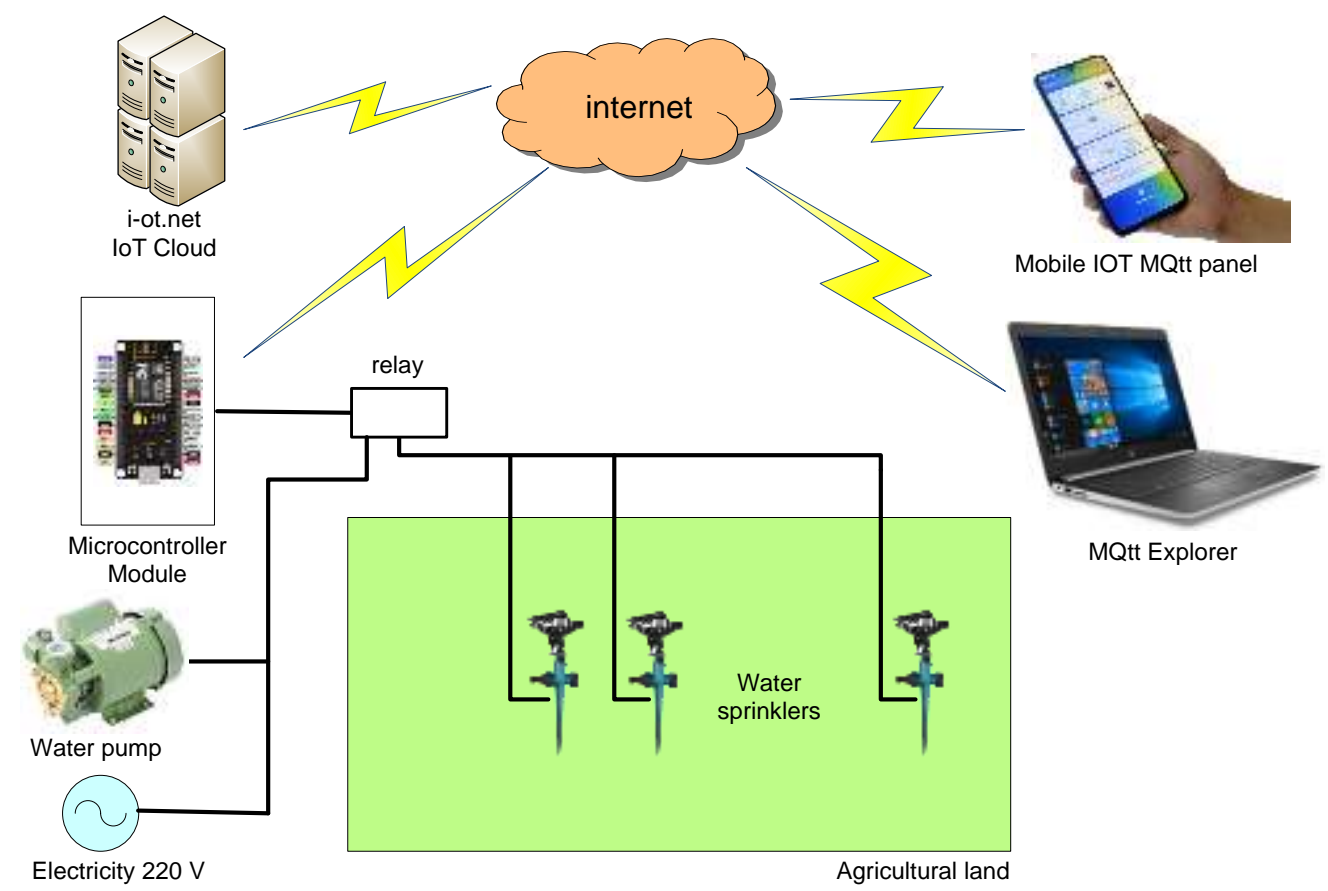

Figure 4. Smart farming

In Figure 3, the Miniature Temperature Control System is designed to be used as a simple temperature monitoring and control solution. The microcontroller module used is NodeMCU. With the supporting circuits, this system is equipped with an LM35 sensor. Based on the reading of this temperature sensor, it is used to decide whether the measured temperature is following the desired temperature value or not. The way to control it is by turning the fan on and off during the startup time as needed. Control can be done automatically, or by pressing a button on the Mo- 
bile Phone via an internet connection. An internet connection must be connected between a microcontroller device that is equipped with an LM35 sensor, the i-ot.net as IoT Cloud, and the IoT MQTT Panel application on the Mobile Phone.

Whereas in Figure-4, Smart Farming is designed to be used as a solution for monitoring and controlling soil quality, $\mathrm{pH}$, temperature, moisture, or others. As in Figure-3, the microcontroller used is NodeMCU. With the supporting circuits, this system is equipped, among others, with a soil moisture sensor. Based on the results of the soil moisture sensor reading, it is used to decide whether agricultural land needs to be watered or not. Watering can be done automatically, or by pressing a button on the Mobile Phone via an internet connection. An internet connection must be connected between a microcontroller device that is equipped with sensors, the i-ot.net as IoT Cloud, and the IoT MQTT Panel application on the Mobile Phone.

\section{Results and Discussion}

Hardware Miniature Temperature Control System as shown in Figure-5. The microcontroller in the circuit, namely NodeMCU, is equipped with an LM35 sensor, equipped with a relay module, power, and a fan which is used to control the temperature. Then for the settings in the IoT MQTT Panel according to the data obtained from the broker or IoT Cloud i-ot.net as shown in Figure-6. Where settings consisting of Connection Name and Client ID can be made arbitrarily, for example, Temperature Control. Broker Web / IP Address to be inputted with i-ot.net. The port number and network protocol are inputted with 1883 and TCP, respectively. Then enter the user and password as given by the broker.

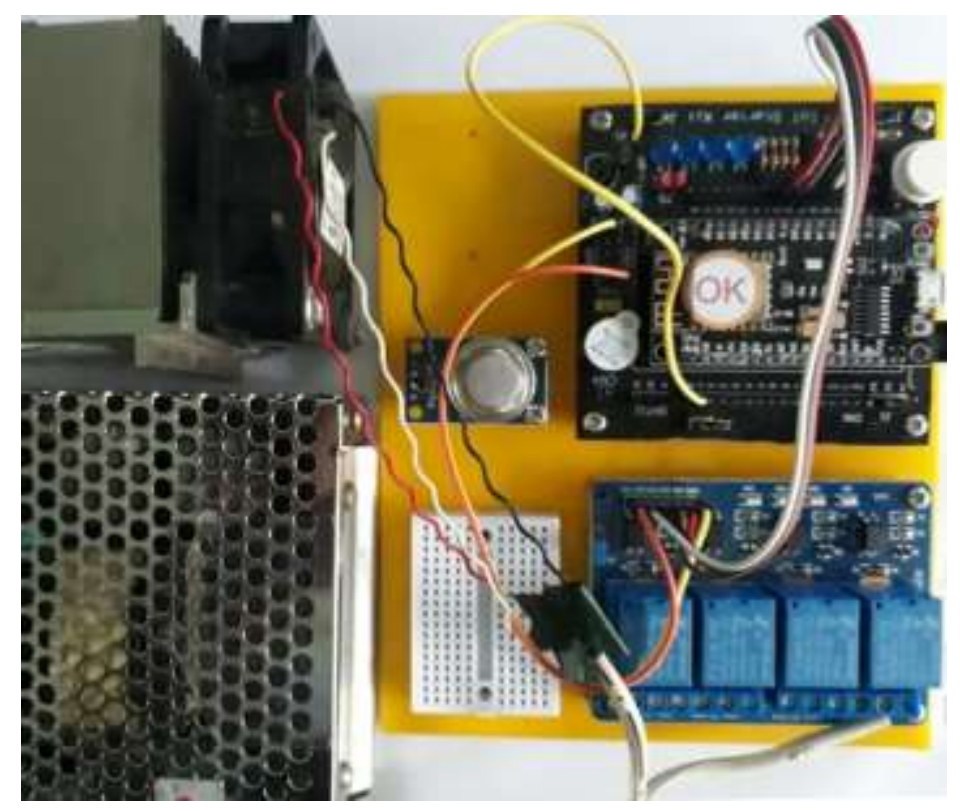

Figure 5. Miniature temperature control system hardware 


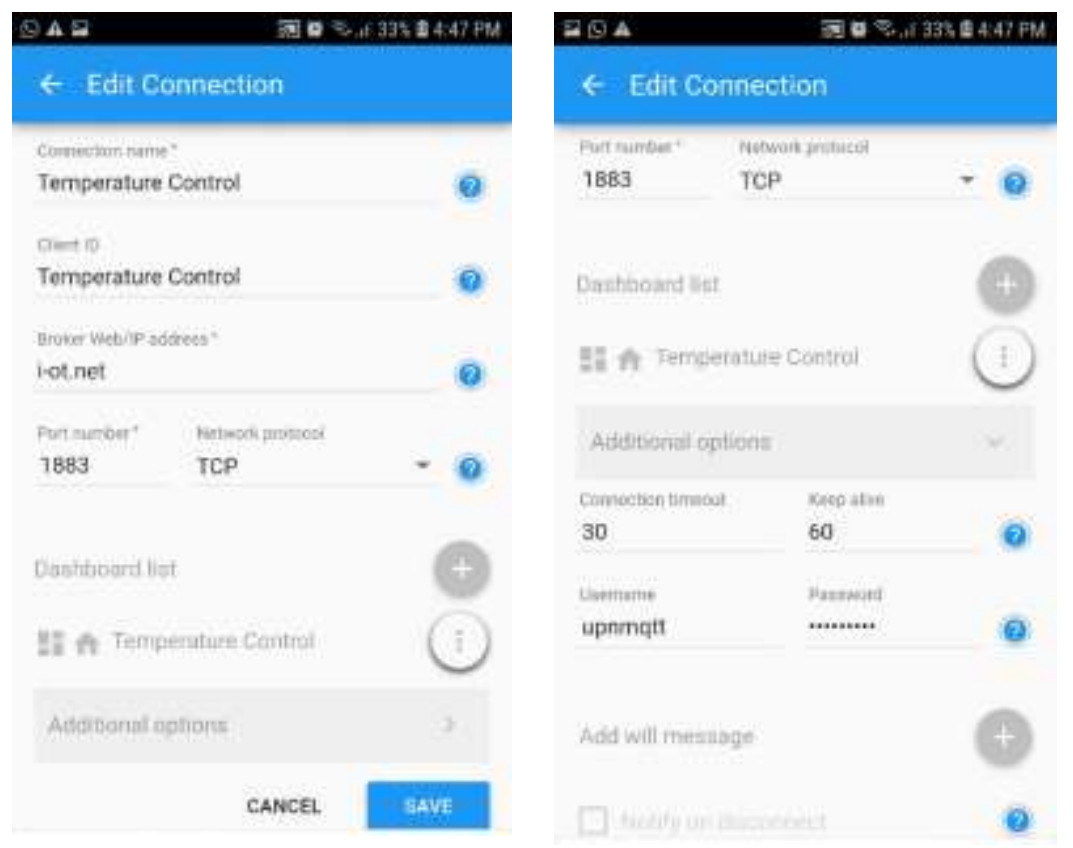

Figure 6. IoT MQTT Panel Settings

Furthermore, the program that must be embedded in the NodeMCU microcontroller is adjusted to the settings provided by the broker or IoT Cloud, as shown in Figure-6. An example of a microcontroller program for a Miniature Temperature Control System, as shown in the following coding.

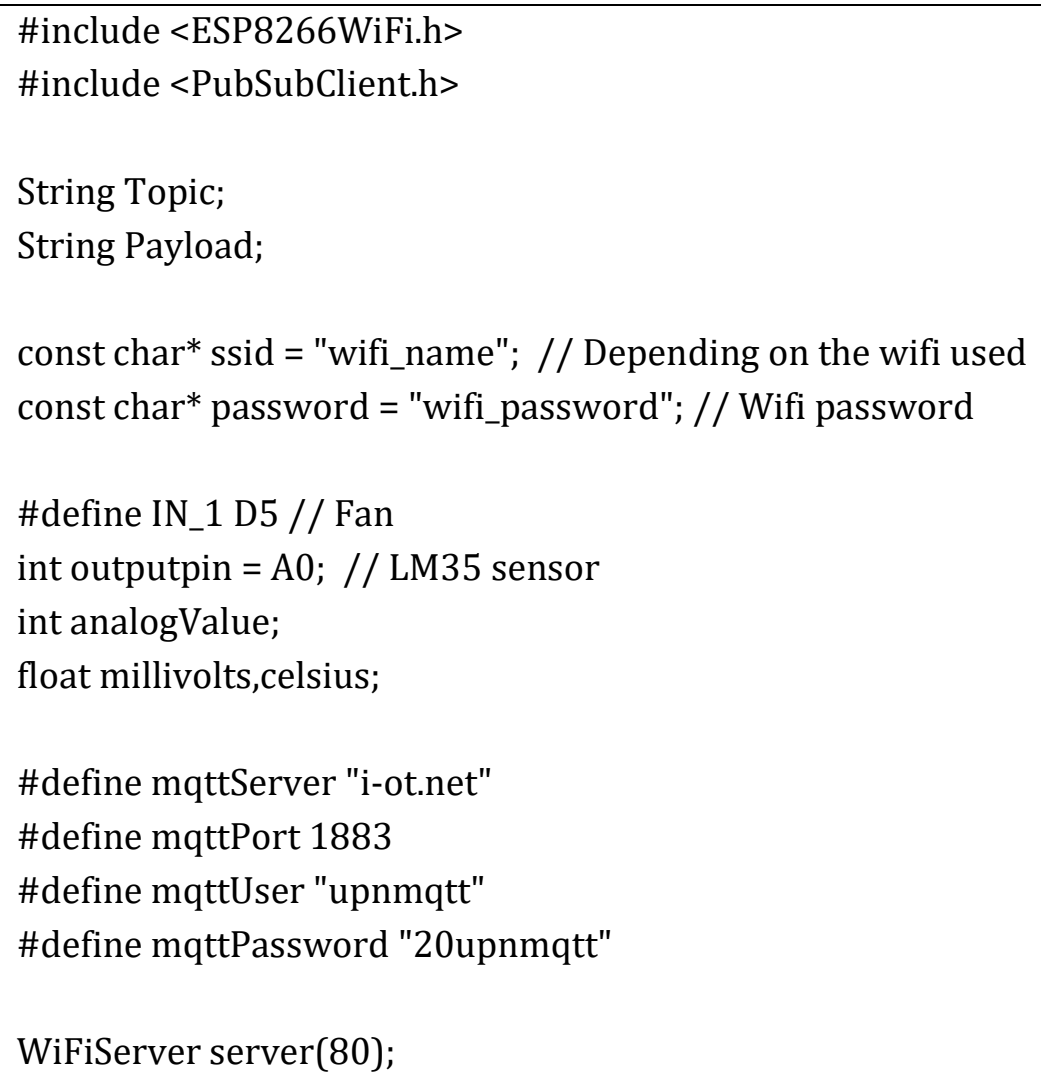




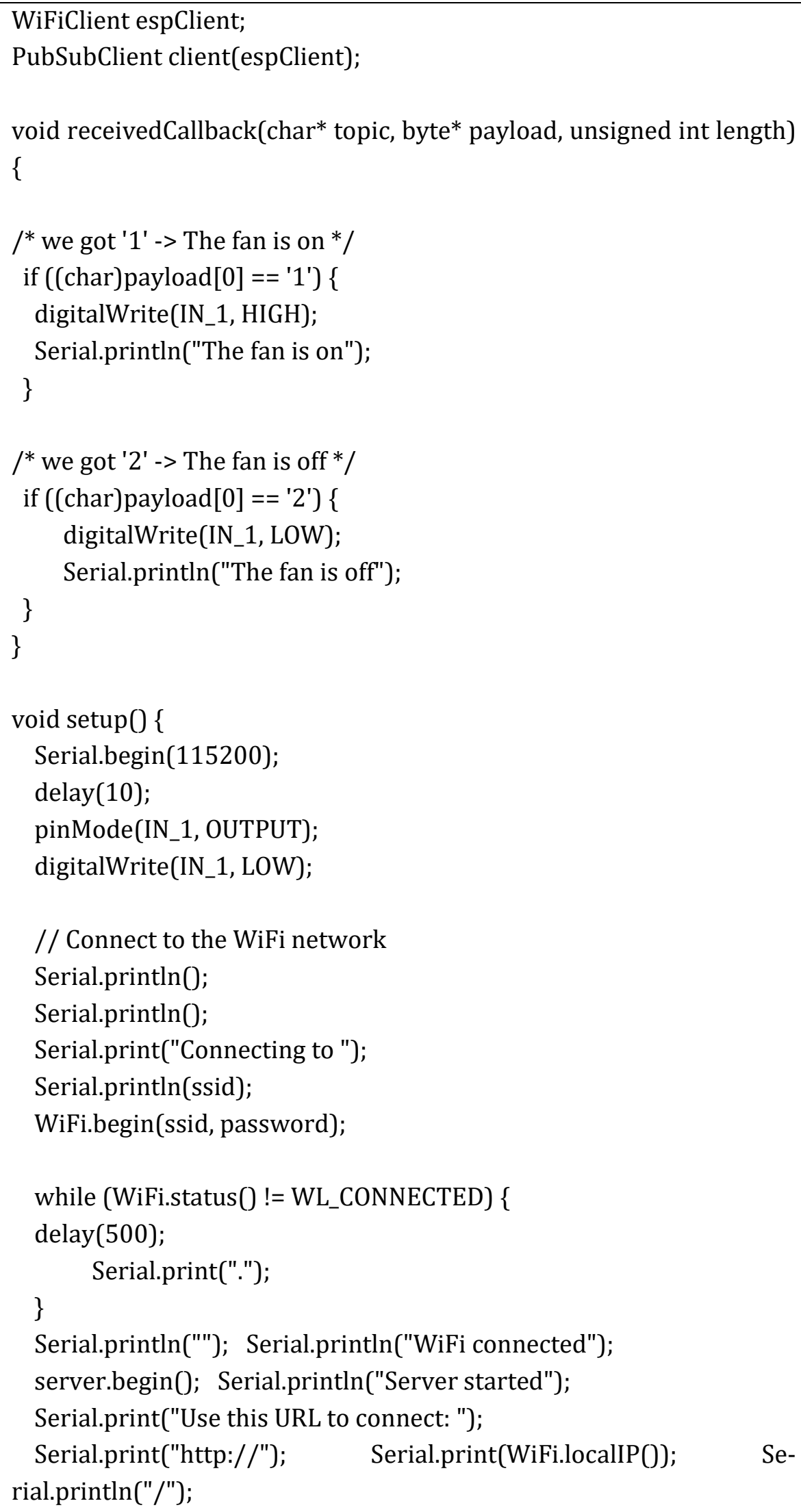




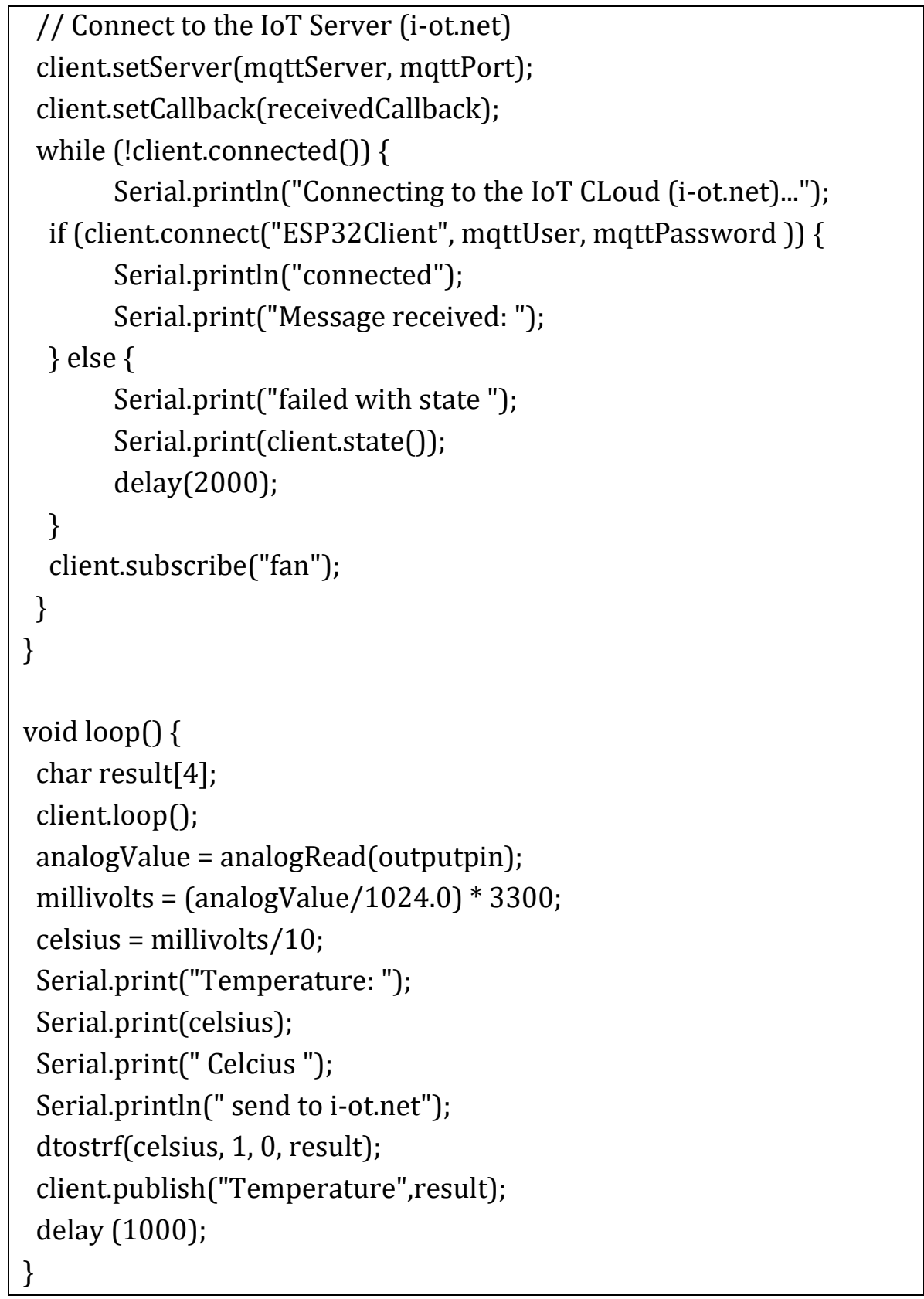

After the compilation and uploading process is carried out to the NodeMCU microcontroller, then testing is carried out. An example of the results of temperature control in the IoT MQTT Panel by turning on and off the fan is shown in Figure 7. 

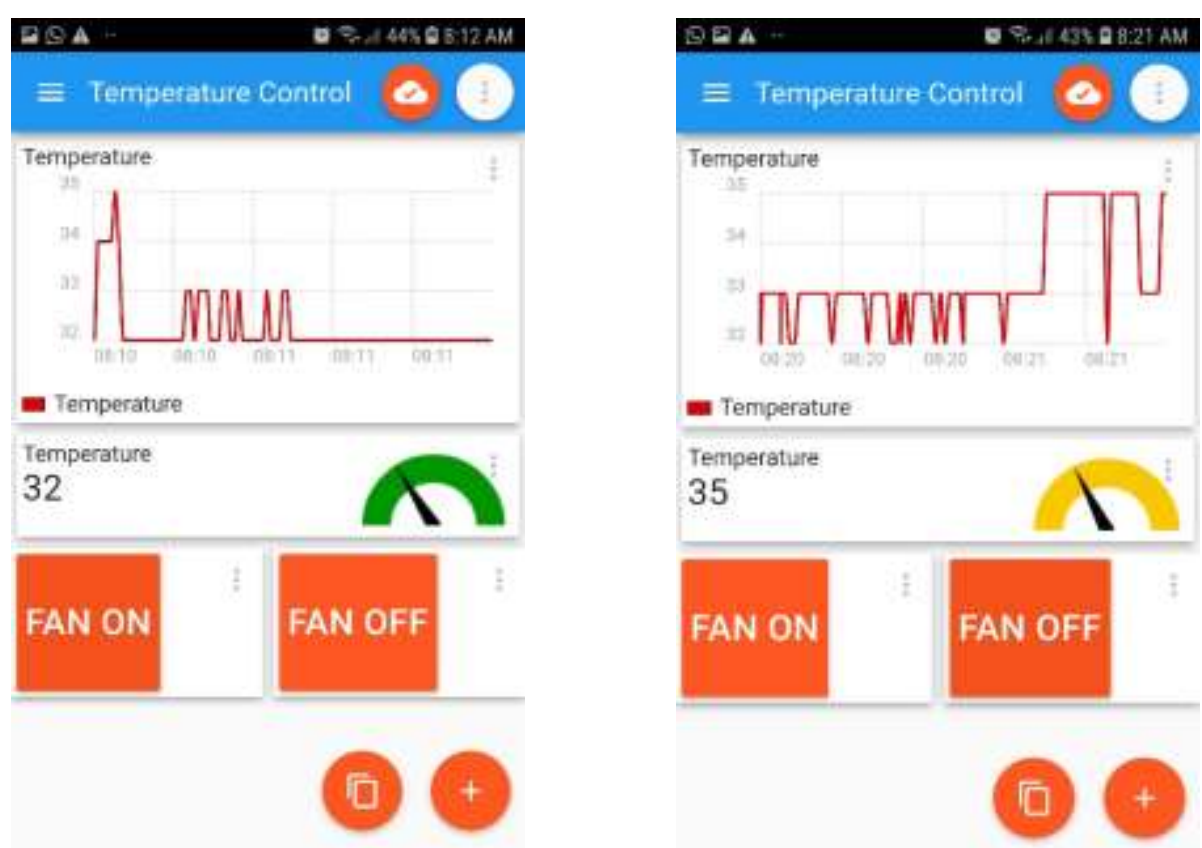

Figure 7. The results of temperature control and monitoring in the IoT MQTT Panel

In the same way as the Miniature Temperature Control System, the Smart Farming system is adjusted. The settings are according to the design in Figure-4. Furthermore, the IoT MQTT Panel is arranged according to the data provided by the broker or IoT Cloud i-ot.net. Likewise, adjustments to the program that must be embedded in the NodeMCU microcontroller are also made, according to the needs of controlling and monitoring soil moisture. Then the system was tested. An example of the results of monitoring and controlling soil moisture is shown in Figure 8.
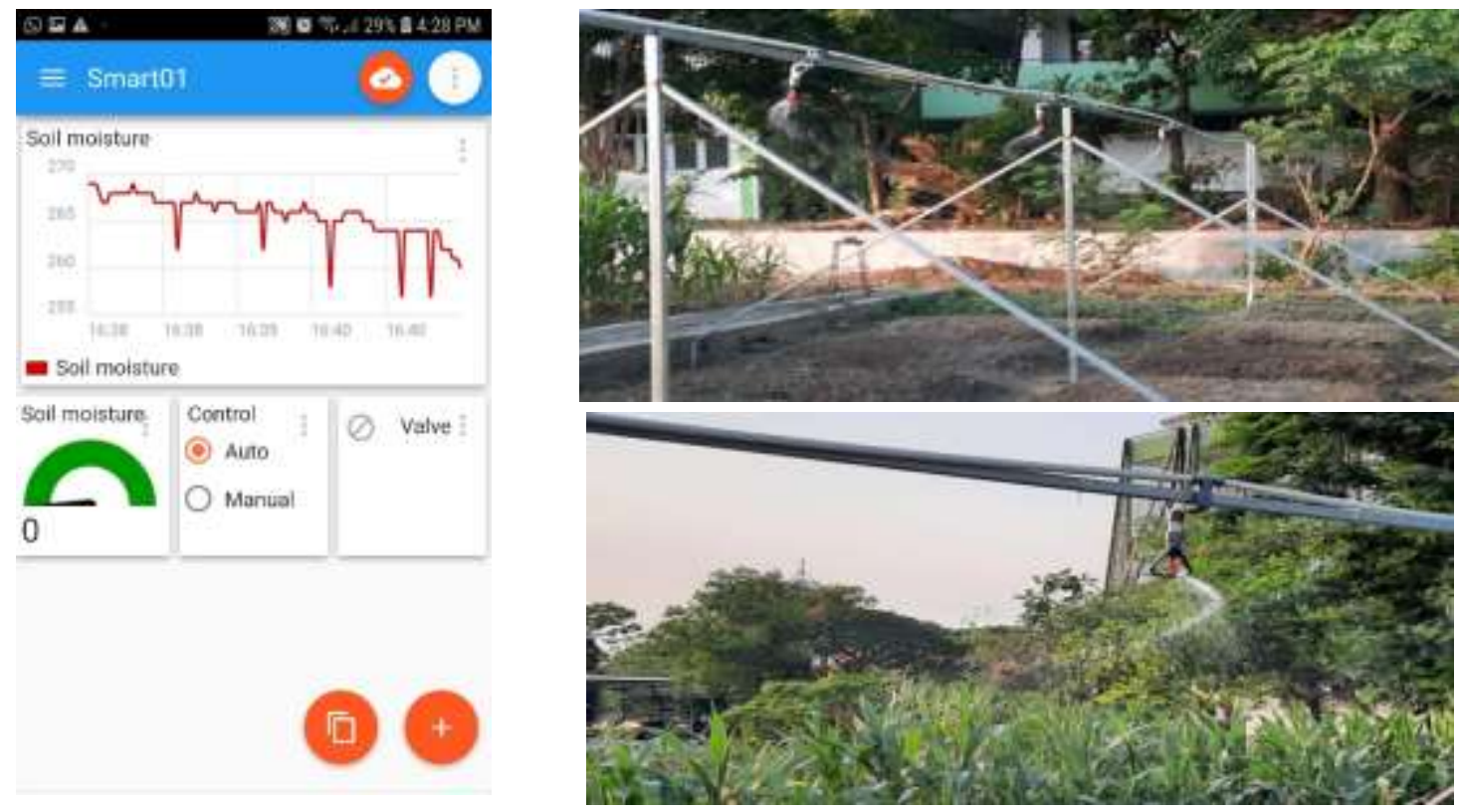

Figure 8. Results of monitoring and controlling soil moisture in smart farming 


\section{Conclusion}

It has been tested, the i-ot.net as the Internet of Things (IoT) Cloud, for monitoring and control of Miniature Temperature Control Systems and Smart Farming. From the test results of both the Miniature Temperature Control System and Smart Farming, monitoring and controlling temperature and soil moisture can be carried out through a Mobile Phone using the IoT MQTT Panel on Android. From the results of this test, the IoT Cloud i-ot.net can then be used widely in Indonesian and international society.

\section{Acknowledgment}

The first author is grateful to the Ministry of Education and Culture, Universitas Pembangunan Nasional "Veteran" Jawa Timur for funding this research based on the Assignment Agreement Letter in the Context of Implementing the Independent Research Program to Improve the Quality of Learning Skim Universitas Pembangunan Nasional "Veteran" Jawa Timur for the 2020 Fiscal Year, Number: SPP / 96 /UN.63.8/LT/VII/2020.

\section{References}

anonymous (2019). mCLOUD IoT platform services. mthinx.com.

Castañeda-Miranda, A., \& Castaño-Meneses, V. M. (2020). Internet of things for smart farming and frost intelligent control in greenhouses. Computers and Electronics in Agriculture, 176, 105614. doi: https://doi.org/10.1016/j.compag.2020.105614.

Chahal, R. K., Kumar, N., \& Batra, S. (2020). Trust management in social Internet of Things: A taxonomy, open issues, and challenges. Computer Communications, 150, 13-46. doi: https://doi.org/10.1016/i.comcom.2019.10.034.

Chen, W. (2020). Intelligent manufacturing production line data monitoring system for industrial internet of things. Computer Communications, 151, 31-41. doi: https://doi.org/10.1016/i.comcom.2019.12.035.

Deperlioglu, O. et al. (2020). Diagnosis of heart diseases by a secure Internet of Health Things system based on Autoencoder Deep Neural Network. Computer Communications, 162, 31-50. doi: https://doi.org/10.1016/j.comcom.2020.08.011.

Feng, X., \& Hu, J. (2020). Research on the identification and management of vehicle behaviour based on Internet of things technology. Computer Communications, 156, 68-76. doi: https://doi.org/10.1016/i.comcom.2020.03.035.

Guan, J. et al. (2020). Robot formation control based on internet of things technology platform. IEEE Access, 8, 96767-96776. doi: 10.1109/ACCESS.2020.2992701.

Huang, X. (2020). Intelligent remote monitoring and manufacturing system of production line based on industrial Internet of Things. Computer Communications, 150, 421-428. doi: https://doi.org/10.1016/j.comcom.2019.12.011.

Iqbal, A. et al. (2018). Interoperable Internet-of-Things platform for smart home system using Web-of-Objects and cloud. Sustainable Cities and Society, 38, 636-646. doi: https://doi.org/10.1016/j.scs.2018.01.044.

Mukherjee, A., De, D., \& Ghosh, S. K. (2020). FogIoHT: A weighted majority game theory based energy-efficient delay-sensitive fog network for internet of health things. Internet of Things, 11, 100181. doi: https://doi.org/10.1016/j.iot.2020.100181.

Muralidhara, S., Hegde, N., \& Rekha, P. M. (2020). An internet of things-based smart energy meter for monitoring device-level consumption of energy. Computers \& Electrical Engineering, 87, $106772 . \quad$ doi: https://doi.org/10.1016/j.compeleceng.2020.106772.

Pinheiro, P. R. et al. (2020). Integration of the mobile robot and internet of things to monitor older people. IEEE Access, 8, 138922138933. doi: 10.1109/ACCESS.2020.3009167.

Ramli, M. R. et al. (2020). IoT-based adaptive network mechanism for reliable smart farm system', Computers and Electronics in Agriculture, 170, 105287. doi: https://doi.org/10.1016/i.compag.2020.105287.

Ravidas, S. et al. (2019). Access control in Internet-of-Things: A survey. Journal of Network and Computer Applications, 144, 79-101. doi: https://doi.org/10.1016/j.jnca.2019.06.017.

Zhang, H., \& Lu, X. (2020). Vehicle communication network in intelligent transportation system based on Internet of Things. Computer Communications, 160, 799-806. doi: https://doi.org/10.1016/j.comcom.2020.03.041. 